\title{
Pathogenesis and treatment of primary aldosteronism
}

\author{
Maria-Christina Zennaro $^{1,2 \dagger}$, Sheerazed Boulkroun $^{1}$ and Fabio L. Fernandes-Rosa ${ }^{1}$ \\ ${ }^{1}$ INSERM, PARCC, Université de Paris, F-75015 Paris, France. \\ ${ }^{2}$ Assistance Publique-Hôpitaux de Paris, Hôpital Européen Georges Pompidou, Service de \\ Génétique, Paris, France. \\ ${ }^{\dagger}$ e-mail: maria-christina.zennaro@inserm.fr
}

\begin{abstract}
Early diagnosis and appropriate treatment of primary aldosteronism, the most frequent cause of secondary hypertension, are crucial to prevent deleterious cardiovascular outcomes. In the last decade, the discovery of genetic abnormalities responsible for sporadic and familial forms of primary aldosteronism has improved the knowledge of the pathogenesis of this disorder. Mutations in genes encoding ion channels and pumps lead to increased cytosolic concentrations of calcium in zona glomerulosa cells, which triggers $C Y P 11 B 2$ expression and autonomous aldosterone production. Improved understanding of the mechanisms underlying the disease is key to improving diagnostics and to developing and implementing targeted treatments. This Review provides an update on the genetic abnormalities associated with sporadic and familiar forms of primary aldosteronism, their frequency among different populations and the mechanisms explaining excessive aldosterone production and adrenal nodule development. The possible effects and uses of these findings for improving the diagnostics for primary aldosteronism are discussed. Furthermore, current treatment options of primary aldosteronism are reviewed, with particular attention to the latest studies on blood pressure and cardiovascular outcomes following medical or surgical treatment. The new perspectives regarding the use of targeted drug therapy for aldosterone-producing adenomas with specific somatic mutations are also addressed.
\end{abstract}




\section{Introduction}

Arterial hypertension is a major cardiovascular risk factor. Among the secondary forms of hypertension, primary aldosteronism is the most frequent form, accounting for approximately $5 \%$ of patients with hypertension in primary care and $10 \%$ to $20 \%$ of patients with hypertension referred to specialist care ${ }^{1-3}$. Primary aldosteronism is caused by autonomous aldosterone production from the adrenal gland, which in the majority of cases is attributable to a unilateral aldosterone producing adenoma (APA) or to bilateral adrenal hyperplasia ${ }^{4}$. The majority of cases of primary aldosteronism are sporadic, while approximately $6 \%$ of patients carry a familial form of the disease ${ }^{5}$. So far, four different Mendelian forms of the disease, transmitted as autosomal dominant traits, have been associated with different genetic defects ${ }^{6-10}$.

Aldosterone production in primary aldosteronism is autonomous from its physiological regulators and inappropriate to the salt and blood volume status of the individual. In these conditions, high aldosterone production leads to blood volume expansion, sodium retention and increased urinary excretion of potassium and hydrogen, which lead to hypertension, hypokalaemia and metabolic alkalosis ${ }^{11}$. Patients with primary aldosteronism are at increased risk of cardiovascular disease compared with patients with essential hypertension matched for age and blood pressure ${ }^{12,13}$. Indeed, increased risk of stroke, myocardial infarction, atrial fibrillation and renal damage has been consistently reported among different studies of patients with primary aldosteronism ${ }^{12,14-17}$. A meta-analysis published in 2018 that compared 3,838 patients with primary aldosteronism and 9,284 patients with essential hypertension reported a statistically significant increased risk of stroke, coronary artery disease, atrial fibrillation, heart failure and left ventricular hypertrophy in the patients with primary aldosteronism ${ }^{13}$. This finding is attributable to the deleterious effects of aldosterone itself, which promotes cardiac and vascular fibrosis and tissue damage independently of blood pressure levels ${ }^{18-21}$. 


\section{Underdiagnosed and hard to diagnose}

The diagnosis of primary aldosteronism is based on an increased aldosterone to renin ratio, calculated from the plasma aldosterone and plasma renin activity or direct plasma renin concentration, at normal dietary salt intake and serum potassium levels and without antihypertensive drugs affecting the renin-angiotensin-aldosterone system. Primary aldosteronism is confirmed with a commonly used confirmatory test (oral sodium loading, saline infusion, fludrocortisone suppression, captopril challenge test, furosemide upright test) followed by subtyping of unilateral versus bilateral forms by adrenal imaging and adrenal vein sampling (AVS) ${ }^{4}$. Primary aldosteronism is found in up to $5 \%$ of patients with hypertension in primary care ${ }^{1,2}$, but its prevalence increases with severity of hypertension and reaches more than $20 \%$ in patients with severe or treatment-resistant hypertension (use of three or more conventional antihypertensive drugs, including a diuretic) ${ }^{3}$

,22. However, only a minority of patients with primary aldosteronismare offered appropriate management and therapeutic options ${ }^{23}$. Simplifying the screening procedures and extending them to all patients with hypertension instead of restricting these procedures to defined categories of patients with severe or resistant hypertension, hypokalaemia or familial hypertension has been suggested and is indeed recommended in certain countries ${ }^{23,24}$. Confirmation of primary aldosteronism requires one or more different confirmatory tests, including oral salt loading or captopril challenge tests ${ }^{4}$. Subtyping unilateral forms of primary aldosteronism (which can be cured with unilateral adrenalectomy), is an extremely important, though difficult, step. AVS is technically challenging and is therefore performed in reference centres only; when performed, protocols and criteria for lateralization differ between centres ${ }^{4}$. Comprehensive profiling of multiple steroids might enable some of these issues to be overcome, in particular enabling the achievement of increased aldosterone lateralization indices in patients with APA and the stratification of APA versus bilateral adrenal hyperplasia on the basis of 
peripheral steroid profiles ${ }^{25,26}$. Published in 2016, an outcome-based randomised diagnostic trial compared CT-based management with AVS-based management to determine the presence of APA (with subsequent treatment consisting of adrenalectomy) or bilateral adrenal hyperplasia (subsequent treatment with mineralocorticoid receptor antagonists (MRAs) ${ }^{27}$. No differences in the daily defined doses of antihypertensive medication required to control blood pressure or in health-related quality of life were found between patients in the CT-based group vs the AVS-based group. Furthermore, biochemical cure was achieved at similar proportion between the two groups. However, results from this study have been widely debated and are still a matter of controversy, particularly considering its limitations with regard to the selection of patients, chosen endpoints and the AVS procedures used ${ }^{28}$. Indeed, a study published in 2018 showed lower complete biochemical success in patients with APA diagnosed only by CT than in those diagnosed using $\mathrm{AVS}^{29}$. In addition, AVS-guided surgery provided a higher rate of cure of hypertension in a large multicentre study that compared the two diagnostic approaches $^{30}$. Further studies are certainly required to minimize the use of AVS given its cost and complexity and more broadly to identify biomarkers that can be used to simplify the diagnostic procedures of primary aldosteronism.

\section{Pathogenesis of primary aldosteronism}

Germline mutations in inherited forms of primary aldosteronism. Two forms of familial hyperaldosteronism $(\mathrm{FH})$ were described several decades ago; FH-I was the first form of FH to be associated with a specific genetic defect and FH-II included all other cases ${ }^{6,31}$. A third form, FH-III, associated with severe hypertension and profound hypokalemia and not attributable to FH-I, was reported in $2008^{32}$. In 2018, whole-exome sequencing studies ${ }^{9,10}$ have identified germline mutations in genes encoding ion channels in a number of patients diagnosed with FHII, which has enabled the reclassification of familial forms of primary aldosteronism according to the underlying genetic defect. 
FH-I (also known as glucocorticoid-remediable aldosteronism) is characterized by severe hypertension in childhood or young adults with autosomal dominant inheritance ${ }^{33,34}$. FH-I is due to a chimeric gene, resulting from an unequal crossing over event that fuses the regulatory regions of $C Y P 11 B 1$, encoding $11 \beta$-hydroxylase, to the coding sequence of $C Y P 11 B 2$, encoding aldosterone synthase ${ }^{6,35}$. These enzymes are responsible for the last steps of cortisol and aldosterone biosynthesis, respectively. Formation of the chimeric gene leads to ectopic expression of $C Y P 11 B 2$ throughout the adrenal cortex, with inappropriate regulation of aldosterone biosynthesis by adrenocorticotropic hormone (ACTH) and aldosterone being also produced in the zona fasciculata ${ }^{36,37}$. In addition, high levels of the hybrid steroids 18oxocortisol and 18-hydroxycortisol are excreted in the urine, due to the expression of aldosterone synthase in the adrenal zona fasciculata and/or zona reticularis ${ }^{38,39}$ (Figure 1).

FH-II is the most common form of familial hyperaldosteronism, with a prevalence of $1.2-6 \%$ in adults with primary aldosteronism ${ }^{5,40-42}$. The phenotypic presentation is variable and indistinguishable from that of primary aldosteronism without familial inheritance. Diagnosis used to be based on the presence of two or more affected family members, after FH-I had been excluded $^{31,40}$. In the past 9 years, the identification of germline mutations in genes encoding ion channels in patients diagnosed with FH-II allowed the reclassification of some patients as having FH-III and FH-IV ${ }^{8,43,44}$ (see later section). In a study published in 2018, a germline mutation in $C L C N 2$, encoding the chloride channel ClC-2, was identified in the first reported FH-II family 9 . The same mutation was found in four other unrelated patients and four additional CLCN2 mutations were identified in unrelated patients with early onset primary aldosteronism ${ }^{9}$. Concomitantly, a de novo germline CLCN2 mutation was identified in a 9-year-old girl with hypertension due to primary aldosteronism ${ }^{10}$. CLCN2 mutations induce a gain of function of 
ClC-2 channels. They induce major changes in channel properties, leading to sustained chloride efflux from the cell. This efflux leads to plasma membrane depolarization, activation of calcium signalling and increased $C Y P 11 B 2$ expression, which increases aldosterone production $^{10}$ (Figure 1). Currently, the term FH-II is used for familial forms of primary aldosteronism that are associated with germline CLCN2 mutations ${ }^{45}$.

FH-III was described as severe early-onset hypertension refractory to medical treatment associated with profound hypokalaemia, high concentrations of the hybrid steroids 18oxocortisol and 18-hydroxycortisol in urine and massive bilateral adrenal hyperplasia ${ }^{32}$. Germline mutations in $K C N J 5$, which encodes the $\mathrm{G}$ protein-activated inward rectifier potassium channel GIRK4, were identified in affected patients ${ }^{7}$ (Figure 1). Variable severity of hyperaldosteronism was observed among FH-III kindreds, which was associated with the type of KCNJ5 mutation in some patients ${ }^{43,46-48}$ but not in others ${ }^{49}$. Investigation of the adrenal glands of two patients with FH-III showed adrenal enlargement, with loss of zonation and expression of aldosterone synthase throughout the adrenal cortex. Remarkably, aldosterone synthase was often co-expressed with $11 \beta$-hydroxylase or $17 \alpha$-hydroxylase, or with both, which explains the increased levels of the hybrid steroids 18-hydroxycortisol and 18-oxocortisol in these patients ${ }^{50}$. In addition, aldosterone and cortisol co-secretion has been observed in one patient carrying a germline $K C N J 5$ mutation $^{51}$.

Germline mutations in $C A C N A 1 H$, which encodes the pore-forming $\alpha 1$ subunit of the T-type voltage-dependent calcium channel Cav3.2, are responsible for FH-IV. A recurrent germline CACNA1H mutation (p.Met1549Val) was identified in five children with primary aldosteronism diagnosed before they were 10 years old, which in two patients was associated with developmental delay or attention deficit disorder ${ }^{8}$. No adrenal abnormality was observed 
on adrenal imaging, but histological examination in one patient showed micronodular adrenal hyperplasia ${ }^{8}$. Additionally, a de novo germline $C A C N A 1 H$ mutation affecting the same codon (p.Met1549Ile) was identified in a patient with early onset primary aldosteronism associated with multiplex development disorder ${ }^{44}$. Germline $C A C N A 1 H$ mutations were also identified in patients with mild primary aldosteronism, previously diagnosed as FH-II, which suggests that CACNA1H might be a susceptibility gene for different forms of the disease ${ }^{44}$ (Figure 1).

Finally, de novo germline mutations in CACNA1D, which encodes Cav1.3 (the voltagedependent L-type calcium channel subunit alpha-1D), were described in two children with a severe early-onset form of hyperaldosteronism that was associated with a complex neurologic disorder (Primary Aldosteronism, Seizures and Neurologic Abnormalities (PASNA)) $)^{52}$. Following the first description, two other cases of PASNA associated with de novo germline CACNA1D mutations were described, one of the patients also presenting congenital hyperinsulinism. ${ }^{53,54}$. In addition, a germline CACNA1D mutation (G403D) previously identified in one patient with PASNA, was identified in a patient with congenital hyperinsulinism, seizures, but without signs of hyperaldosteronism ${ }^{55}$, suggesting that PASNA is a complex disease with different clinical manifestations.

Somatic mutations in aldosterone producing adenomas. In most cases, lateralized aldosterone production is attributable to an APA, which can be a unique nodule or found within a multinodular adrenal gland ${ }^{56-59}$. In some cases, AVS identifies lateralized aldosterone production in adrenal glands without nodules observed at imaging, which is associated with the presence of micronodules or unilateral adrenal hyperplasia ${ }^{60}$. In APA, somatic mutations are found in genes associated with familial forms of primary aldosteronism, $\mathrm{KCNJ}^{7}$ and $C A C N A 1 D^{52,61}$, as well as in genes encoding ATPases, ATP1Al and ATP2B361,62. The most 
frequent genetic abnormalities are recurrent mutations in $K C N J 5$, which are found in more than $40 \%$ of $\mathrm{APA}^{63}$, ranging between $12 \%$ and $80 \%$ across different studies, with higher frequencies in patients from $\mathrm{Asia}^{64}$. CACNA1D mutations are found in up to $10 \%$ of patients with APA, while $A T P 1 A 1$ and $A T P 2 B 3$ are less frequent ${ }^{65}$.

In addition to these genes, somatic mutations in $C T N N B 1$ have been described in $2-5 \%$ of patients with $\mathrm{APA}^{52,66,67}$, and an association with pregnancy or menopause was suggested ${ }^{68}$. Somatic mutations in PRKACA have been described in rare cases ${ }^{69}$. As these mutations are similar to those found in cortisol producing adenoma and adrenocortical cancer ${ }^{70-75}$, their specific role in APA development remains an open question.

Studies published in the past 3 years that performed genetic testing on adrenal nodules expressing aldosterone synthase using immunohistochemistry-guided next-generation sequencing (NGS) show that somatic mutations in APA driver genes are found in $88-90 \%$ of patients $^{76-78}$. The increased detection of mutations when compared with studies performing Sanger sequencing in DNA extracted from frozen APA is attributable to improved identification of aldosterone producing structures in multinodular adrenals with APA, as well as improved sensitivity and coverage of candidate genes with NGS. Aldosterone synthase immunohistochemistry-guided NGS also showed that somatic CACNA1D mutations are the most frequent genetic event found in APA from African American patients ${ }^{77}$.

Somatic mutations were also identified in micronodules in adrenal glands from patients with lateralized primary aldosteronism without $\mathrm{APA}^{60}$ and in different secondary nodules from patients with multinodular adrenal glands ${ }^{56,59,78}$. In these cases, different micronodules of the same adrenal might have different mutations. Other adrenal structures with somatic mutations are the aldosterone producing cell clusters (APCC, discussed later), which are groups of 
aldosterone synthase expressing cells found in normal adrenals that are supposed to autonomously produce aldosterone ${ }^{79}$. Somatic mutations are found in CACNA1D and ATP1AI in APCC from normal adrenals ${ }^{79}$. In a study published in 2020, KCNJ5 mutations were also observed in APCC from different adrenals with an $\mathrm{APA}^{78}$, which suggests that $K C N J 5$ mutations in APCC could be more prevalent than previously observed. In addition, an increase in the number or enlargement of APCC with somatic mutations in CACNA1D and a KCNJ5 mutation in a micro-APA has been reported in patients with bilateral adrenal hyperplasia ${ }^{80}$.

Mechanisms leading to autonomous aldosterone production. Aldosterone is synthesized from cholesterol in the zona glomerulosa of the adrenal cortex through different enzymatic reactions that are catalyzed by cytochrome P450 enzymes and hydroxysteroid dehydrogenases. The last steps in aldosterone biosynthesis are catalyzed by aldosterone synthase, encoded by CYP11B2, which is an enzyme specifically expressed in the zona glomerulosa. In zona glomerulosa cells, activation of $\mathrm{Ca}^{2+}$ signalling, following stimulation by angiotensin II or an increase in extracellular concentrations of potassium, is the major trigger for aldosterone biosynthesis ${ }^{81}$.

All mutations affecting ion channels and pumps identified in primary aldosteronism lead to increased intracellular concentrations of $\mathrm{Ca}^{2+}$ and autonomous aldosterone production, but the underlying mechanisms leading to activation of $\mathrm{Ca}^{2+}$ signalling are different for each type of channel and pump. Schematically, mutations affecting GIRK4, the $\alpha 1$ subunit of the $\mathrm{Na}^{+}, \mathrm{K}^{+}-$ ATPase or CLC-2, all induce cell membrane depolarization, followed by opening of voltagegated $\mathrm{Ca}^{2+}$ channels and increased intracellular concentrations of $\mathrm{Ca}^{2+}$. By contrast, mutations in PMCA3, Cav1.3 and Cav3.2 directly increase intracellular levels of $\mathrm{Ca}^{2+}$.

GIRK4 is a $\mathrm{G}$ protein-activated inward rectifier potassium channel composed of two transmembrane domains (M1 and M2), a pore region (H5) and an intracellular N-terminus and 
C-terminus (Figure 1). Mutations in GIRK4 localize near or within the selectivity filter (p.Gly151Arg, p.Thr158Ala and p.Leu168Arg) and induce a change in the ion selectivity of the channel $^{7}$. In addition to the loss of $\mathrm{K}^{+}$selectivity, the mutated channels gain permeability to sodium, inducing cell membrane depolarization, opening of voltage-gated $\mathrm{Ca}^{2+}$ channels, stimulation of $\mathrm{Ca}^{2+}$ signalling and ultimately increased $C Y P 11 B 2$ expression and aldosterone biosynthesis $^{7,82,83}$. Some mutations localized far away from the selectivity filter (p.Arg115Trp and p.Glu246Gly) do not change the channel selectivity but rather decrease the abundance of the mutated channels at the membrane ${ }^{84}$. An increased degradation of mutant channels in the cytoplasm causing a decrease in GIRK4 expression has been suggested to be the mechanism responsible for aldosterone overproduction and adenoma development ${ }^{84}$. Although in vitro studies have shown no changes in aldosterone production when GIRK4 expression is downregulated by short hairpin RNA in HAC15 cells (a human adrenocortical carcinoma cell line $)^{82}$, it has been suggested that effects of $K C N J 5$ mutations on cell growth may be determined by the expression level of the mutated GIRK4 channel ${ }^{85}$.

CLCN2 encodes ClC-2, a voltage-gated chloride channel composed of 18 transmembrane helices and an intracellular $\mathrm{N}$-terminus and C-terminus ${ }^{86}$ that forms dimers (Figure 1). ClC-2 mutations are localized in different regions of the protein, in so-called 'inactivation domains' (p.Tyr26Asn, p.Gly24Asp, p.Met22Lys and p.Lys362del), or in regions of unknown function (p.Arg172Gly and p.Ser865Arg) $)^{9,10,87}$. ClC-2 mutations induce a loss of the voltage-gating of the channel, leading to increased chloride currents at resting potential. This change results in cell membrane depolarization, followed by opening of voltage-gated calcium channels, and to increased aldosterone biosynthesis through activation of $\mathrm{Ca}^{2+}$ signalling ${ }^{10}$. In addition to germline mutations in FH-II, a somatic CLCN2 mutation was described in one case of APA ${ }^{88}$. In the last year, two mouse models have been described, which recapitulate the human disease 
induced by $C L C N 2$ mutations. Deletion of eight amino acids in the $\mathrm{N}$-terminal region of mouse ClC-2 leads to increased chloride conductance in zona glomerulosa cells, cell membrane depolarization and increased intracellular concentrations of $\mathrm{Ca}^{2+}$. Homozygous mice have high serum levels of aldosterone in the presence of low renin activity, marked hypertension and hypokalaemia ${ }^{89}$. Similarly, heterozygous mice carrying a missense mutation homologous to the most common CLCN2 mutation associated with FH-II exhibited a mild form of primary aldosteronism associated with increased chloride efflux and zona glomerulosa cell membrane depolarization $^{90}$. These two animal models support the role of $\mathrm{ClC}-2$ in zona glomerulosa pathophysiology and represent new valuable tools for the study of primary aldosteronism.

Mutations in the $\alpha 1$ subunit of the $\mathrm{Na}^{+}, \mathrm{K}^{+}$-ATPase also lead to cell membrane depolarization due to loss of pump activity and reduced affinity for potassium ${ }^{61,62}$. Moreover, disturbed $\mathrm{pH}$ homeostasis has been reported for some mutations (p.Leu104Arg, p.Val332Gly) ${ }^{91}$. The $\mathrm{Na}^{+}, \mathrm{K}^{+}-$ ATPase is a member of the P-type ATPase family and is composed of two subunits ( $\alpha$ and $\beta$ ). Four isoforms of the $\alpha$ subunit have been described, and they are composed of 10 transmembrane domains (M1 to M10) and an intracellular N-terminus and C-terminus. The $\mathrm{Na}^{+}, \mathrm{K}^{+}$-ATPase allows exchange of three cytoplasmic sodium ions for two extracellular potassium ions $^{92}$. The mutations identified in the $\alpha 1$ subunit are localized in the $\mathrm{M} 1^{62,93-95}$ and M4 domains ${ }^{61,62}$; interestingly, these two domains have a crucial role in $\mathrm{K}^{+}$binding and gating ${ }^{96}$. Mutations of the $\mathrm{Na}^{+}, \mathrm{K}^{+}$-ATPase depolarize adrenocortical cells, disturb the $\mathrm{K}^{+}$sensitivity and reduce the intracellular $\mathrm{pH}$ level; however, in the human adrenocortical cell line H295R they do not induce an overt increase of intracellular levels of $\mathrm{Ca}^{2+}$, suggesting that increased aldosterone production might result from a combination of different signalling abnormalities ${ }^{91}$. 
Mutations affecting calcium channels (Cav1.3 and Cav3.2) and the plasma membrane calcium ATPase 3 (PMCA3) directly increase the intracellular concentration of $\mathrm{Ca}^{2+}$. Similarly to $\mathrm{Na}^{+}, \mathrm{K}^{+}$-ATPase, PMCA3 belongs to the P-type ATPase family. It is composed of 10 transmembrane domains (M1 to M10) and an intracellular N-terminus and C-terminus. All the mutations in PMCA3 that have been identified in APA are in frame deletions of amino acids localized in the M4 domain between amino acids 422 and 433,62,63,94,95,97. Comparison of PMCA3 with the structure of the sarcoplasmic reticulum type $\mathrm{Ca}^{2+}$-ATPase (SERCA) suggested that mutated amino acids could influence the ATP-driven conformational transition of the pump, modulating $\mathrm{Ca}^{2+}$ occlusion and transport ${ }^{98}$, leading to a reduced capacity of the cells to extrude $\mathrm{Ca}^{2+}$, thus increasing the intracellular concentration of $\mathrm{Ca}^{2+}$. It has also been suggested that, in addition to the loss of the physiological pump function, ATP2B3 mutations could be responsible for $\mathrm{Na}^{+}$and possibly $\mathrm{Ca}^{2+}$ leak into the cell, inducing cell depolarization and thus contributing, via this additional mechanism, to increased intracellular $\mathrm{Ca}^{2+}$ concentrations $^{98}$.

CACNA1D encodes the $\alpha 1$ subunit of the L-type $\mathrm{Ca}^{2+}$ channel Cav1.3 and CACNA1H encodes the $\alpha 1$ subunit of the T-type $\mathrm{Ca}^{2+}$ channel Cav3.2. They are both composed of four repeat domains (I to IV), each consisting of six transmembrane segments ( $\mathrm{S} 1$ to S6), and mediate $\mathrm{Ca}^{2+}$ influx into the cell upon membrane depolarization. Mutations in the Cav1.3 channel affect highly conserved amino acids in different regions of the protein, which have been associated with different channel functions ${ }^{52,61}$. In particular, mutations shift the voltage-dependence of the channels towards more negative potentials or are responsible for delayed voltage-dependent inactivation of the channel, resulting in increased $\mathrm{Ca}^{2+}$ entry into the cells, followed by activation of $\mathrm{Ca}^{2+}$ signalling and increased aldosterone production. Similarly, mutations in Cav3.2 affect different domains and are responsible for alterations of different channel 
properties, including activation, deactivation and inactivation, leading again to increased $\mathrm{Ca}^{2+}$ entry into the cells ${ }^{8,44}$ (Figure 1).

\section{Development of APA and APCC}

Although the mechanisms responsible for autonomous aldosterone production have been clearly established during the past decade, those responsible for APA development are less well characterized. Two hypotheses for APA development have emerged in the past few years, the APCC model and the two-hit model.

The APCC model is based on the idea that mutations in one of the APA driver genes could occur in some zona glomerulosa cells, leading to the formation of APCC that might then develop into possible APCC-to-APA translational lesions (pAATL, discussed later), which could ultimately lead to APA development ${ }^{99}$. APCC are aldosterone producing structures present in normal adrenals as well as in adrenal tissue adjacent to APA or in adrenals from patients with idiopathic hyperaldosteronism ${ }^{80,100,101}$. Next-generation sequencing of DNA isolated from 23 APCC from 11 healthy adrenal glands identified CACNA1D mutations in eight APCC and ATP1Al mutations in two ${ }^{79}$. No mutations in $K C N J 5$, the most frequent alteration observed in APA, were identified in normal adrenals ${ }^{79}$; however, these mutations can be found in APCC from adrenals with APA $^{78}$. Analysis of adrenal glands from 15 patients with idiopathic hyperaldosteronism discovered an accumulation of APCC in comparison with healthy adrenals. CACNA1D mutations were found in 59\% of APCC from adrenals of patients with idiopathic hyperaldosteronism; $33 \%$ of micro-APA also carried a somatic mutation, including one $K C N J 5$ mutation $^{80}$. In addition to APCC, a study published in 2017 described intermediate structures between APCC and APA called pAATL ${ }^{102}$. These structures, consisting of a subcapsular APCC-like part and an inner micro-APA-like part, carry different types of somatic mutations 
in the different parts of the structure, including KCNJ5 mutations ${ }^{99}$. These results suggest that APA could derive from APCC, although the sequence of events leading to the transition of zona glomerulosa cells to APA through APCC and pAATL remains to be established. This hypothesis is also supported by evidence showing that a subgroup of APCC has a metabolic profile similar to that of $\mathrm{APA}^{103}$.

The two-hit model is based on the concept of two consecutive events leading to APA development, with the first event leading to abnormal cell proliferation that creates a propitious environment for the occurrence of somatic mutations in APA driver genes. Adrenals with APA are characterized by adrenal cortex remodelling, reduced vascularization and zona glomerulosa hyperplasia $^{101}$. At the molecular level, these changes are associated with activation of WNT- $\beta$ catenin signalling in the entire adrenal cortex and in two-thirds of APA ${ }^{104,105}$. Resected adrenals often show multiple micronodules in addition to APA, with some of these micronodules expressing aldosterone synthase ${ }^{56,101,106}$. Remarkably, different mutations in APA driver genes were identified in different micronodules from the same adrenal gland ${ }^{56,59}$, which suggests that these mutations occur in a previously remodelled adrenal gland. Reported in 2016, the case of a young patient with lateralized primary aldosteronism and macronodular adrenal hyperplasia further supported a two hit model ${ }^{107}$. The patient carried a germline heterozygous $A P C$ mutation that was associated with $A P C$ inactivation in all nodules of the resected adrenal, whereas a KCNJ5 mutation was identified exclusively in the aldosterone synthase positive nodule ${ }^{107}$. These data suggest that the APC mutation had induced adrenal cortex remodelling and nodule formation, while the KCNJ5 mutation was driving the hormonal secretory pattern.

\section{New diagnostic opportunities}


Genetic diagnosis in familial forms of primary aldosteronism might enable targeted treatment in mutation carriers and improved management of affected family members. In particular, $\mathrm{FH}-$ I can lead to left ventricular abnormalities well before the onset of hypertension ${ }^{108}$. In patients with APA, the identification of any surrogate biomarker of somatic mutations would enable the identification of patients who are suitable candidates for AVS, which would simplify the current diagnostic procedures for a large proportion of patients with low probability of having an APA 109,110

The correlation between the mutation status and particular clinical, biochemical or histological features has been investigated in many different studies. It is generally accepted that $K C N J 5$ mutations are more prevalent in women ${ }^{64,65}$, and patients with younger age, higher aldosterone levels and larger tumours than in patients without these mutations ${ }^{64}$. Other associations, including with the lateralization index at AVS, have been reported ${ }^{94,111}$, but are not replicated across studies ${ }^{112}$. Mutations in CACNA1D, ATP1A1 and ATP2B3 are mainly associated with male sex and smaller tumours ${ }^{52,61-63,66,95,113}$, while CTNNB1 mutations seem to be more prevalent in female patients ${ }^{52,66-68}$.

In the past few years, steroid profiling has enabled the identification of a 7-steroid fingerprint that can be used to correctly classify $92 \%$ of APA according to genotype in peripheral venous plasma $^{111}$. In particular, levels of the hybrid steroid 18-oxocortisol were considerably higher in adrenal and peripheral venous samples from patients with $K C N J 5$ mutations than in samples from other groups ${ }^{111}$, an association that was confirmed by other studies ${ }^{78,114-116}$. These steroid biomarkers, together with peculiar computed tomography imaging characteristics of APA carrying KCNJ5 mutations (larger tumour size and lower pre-contrast Hounsfield units) compared with tumours carrying mutations in other genes ${ }^{66}$, could guide clinical assessment 
towards subtype diagnosis for surgery or targeted medical treatment. Another approach is to use the peculiar pharmacological characteristics of mutated proteins to identify APA carrying somatic mutations to select patients for AVS. In this context, an ongoing study is evaluating the acute changes of plasma concentrations of aldosterone and renin in peripheral venous blood of patients with primary aldosteronism after roxithromycin administration, which specifically blocks mutated GIRK4 channels ${ }^{117}$. It is hypothesized that aldosterone levels will fall after macrolide treatment in patients with APA carrying KCNJ5 mutations, allowing simplified diagnosis and treatment of those patients.

\section{Treatment of primary aldosteronism}

Current treatments and outcomes for primary aldosteronism. Surgical adrenalectomy in lateralized primary aldosteronism or therapy with MRA in bilateral forms are the recommended treatments for primary aldosteronism ${ }^{4}$. The goal of primary aldosteronism treatment is the normalization (or improvement) of blood pressure and correction of hypokalaemia. However, different studies have shown that patients with primary aldosteronism are at increased risk of cardiovascular complications independently of blood pressure levels $\mathrm{s}^{12,14,118-120}$. A meta-analysis of studies comparing cardiovascular events and target organ damage in patients with primary aldosteronism and essential hypertension confirmed an increased risk of stroke, coronary artery disease, atrial fibrillation, heart failure, type 2 diabetes mellitus and left ventricular hypertrophy ${ }^{13}$. Thus, treatment of primary aldosteronism should not just be targeted at blood pressure control but also at decreasing aldosterone production or efficiently blocking the mineralocorticoid receptor (MR). The Endocrine Society Guidelines for primary aldosteronism management recommend unilateral laparoscopic adrenalectomy for patients with unilateral primary aldosteronism or treatment with an MRA for patients unable or unwilling to undergo surgery. For patients with bilateral disease, MRA is recommended ${ }^{4}$. 
Different studies have described an improvement in hypertension after adrenalectomy ${ }^{121-124}$, and a large longitudinal study has shown that adrenalectomy for unilateral primary aldosteronism reduces all-cause mortality compared with MRA treatment alone ${ }^{120}$. After adrenalectomy, patients with APA exhibit a reduced risk of incident stroke after surgery ${ }^{125}$, a regression of left ventricular hypertrophy ${ }^{123}$ and decreased risk of atrial fibrillation after a median follow-up of 11.8 years ${ }^{126}$. In addition, a prospective study performed in 20 patients with APA by the TAIPAI study group highlighted an improvement in myocardial fibrosis, intima-media thickness and arterial stiffness after surgery ${ }^{127,128}$. Surgical treatment for APA was also associated with improved metabolic outcomes and increased insulin sensitivity ${ }^{129}$.

Primary aldosteronism is associated with reduced quality of life and psychological symptoms, with women being more affected than men ${ }^{121,130,131}$. An improvement in quality of life was observed in different studies following treatment, more so after adrenalectomy than in patients receiving MRA $^{30,121,132-134}$. In 2019, a disease-specific quality of life questionnaire for primary aldosteronism was developed and validated for use in clinical practice ${ }^{135}$. This questionnaire may allow a better diagnosis and care of psychological issues related to primary aldosteronism, helping physicians to inform and identify potential cases of depressive disorders associated with the disease.

An international panel of 31 experts from 28 centres were involved in the Primary Aldosteronism Surgical Outcome (PASO) study, which has achieved consensus in developing criteria for classifying surgical outcome and follow-up of patients with unilateral primary aldosteronism $^{124}$. They proposed clinical (blood pressure and use of anti-hypertensive drugs) and biochemical (plasma levels of potassium, aldosterone concentration and concentration or activity of renin in plasma) parameters for classifying complete, partial or absent surgical 
success. The analysis of clinical and biochemical data from $\sim 700$ patients with primary aldosteronism who had undergone adrenalectomy showed that $94 \%$ achieved biochemical cure (normalization of potassium levels and the aldosterone-to-renin ratio), while normalization of blood pressure without the use of anti-hypertensive drugs was achieved in only $37 \%$ of patients ${ }^{124}$.

The use of standardized definitions and nomenclature for APA outcomes is of great importance for the comparison of different cohorts of patients and to identify factors that influence the success of adrenalectomy. Using the PASO criteria, findings from previous studies showing that younger patients and women are more likely to have a favourable surgical outcome ${ }^{122}$ have been clearly confirmed in the PASO multicentre study ${ }^{124}$. Similar results were obtained in a large multicentre study evaluating the outcomes of 1,625 patients undergoing AVS for primary aldosteronism subtyping. Hypertension was cured after adrenalectomy in $19.6 \%$ of the patients, with women showing a more favourable outcome than men $^{30}$. Finally, the evaluation of 574 patients with primary aldosteronism undergoing surgery within the Japanese Nationwide Cohort highlighted five variables as independent predictors for blood pressure normalization: $\leq 7$ years of hypertension, BMI $\leq 25 \mathrm{~kg} / \mathrm{m}^{2}$, no more than one antihypertensive medication, absence of medical history of type 2 diabetes mellitus and female $\operatorname{sex}^{136}$. Female sex, duration of hypertension and lower number of antihypertensive agents were also predictors of hypertension improvement after adrenalectomy in Brazilian patients with primary aldosteronism $^{137}$. Interestingly, in a multivariate analysis, only the presence of a somatic $K C N J 5$ mutation was an independent predictor of blood pressure normalization ${ }^{137}$.

Lifelong MRA therapy, the recommended medical option for patients with bilateral primary aldosteronism and patients with unilateral primary aldosteronism unable or unwilling to 
undergo surgery, improves hypertension in patients with primary aldosteronism ${ }^{138,139}$. Spironolactone is the MRA of choice at the highest tolerable dose that enables control of blood pressure and hypokalaemia ${ }^{23,140}$. The adverse effects of spironolactone include gynecomastia, decreased libido and erectile dysfunction in men, and menstrual irregularity in women ${ }^{141}$. The MRA eplerenone has higher MR selectivity than spironolactone and is therefore associated with fewer adverse effects ${ }^{142,143}$. However, it is less potent than spironolactone and is not approved for treatment of primary aldosteronism in the USA and Europe. Third-generation, non-steroidal MRAs, which are more specific than spironolactone and more potent than eplerenone, are currently under development for the treatment of heart failure and chronic kidney disease $\mathrm{e}^{144}$ and might provide new therapeutic options in primary aldosteronism.

Different studies have tested the efficacy of MRAs for reducing cardiometabolic risks, with some of them suggesting that MRAs might not be effective ${ }^{14,118,119,123,145}$. A large cohort study of 602 patients with primary aldosteronism receiving MRA therapy compared with 41,853 patients with essential hypertension has shown an increase in cardiovascular events, type 2 diabetes mellitus risk and mortality in medically treated patients with primary aldosteronism ${ }^{146}$. Interestingly, the excess risk of cardiovascular events and mortality was observed in patients with primary aldosteronism whose renin activity remained suppressed, but not in those who normalized their renin levels ${ }^{146}$. Accordingly, an increase in atrial fibrillation events was also observed in patients with primary aldosteronism treated with MRA who had suppressed renin activity, in comparison with medically treated patients with unsuppressed renin and patients treated by adrenalectomy ${ }^{147}$. In addition, patients with primary aldosteronism receiving MRA had a higher risk of developing chronic kidney disease than patients undergoing adrenalectomy or patients with essential hypertension ${ }^{148}$. Based on these findings, it was suggested that suppressed renin activity, which is associated with an increase in cardiovascular risk, might be 
a biomarker of incomplete MR blockade. In addition, a medical-surgical approach has been proposed that combines unilateral adrenalectomy to attenuate the severity of primary aldosteronism followed by lifelong MRA therapy in patients with bilateral but asymmetric aldosterone production. This combined approach could be particularly useful in young patients, patients with cardiovascular comorbidities, chronic kidney disease or with persistently elevated blood pressure despite high doses of MRA ${ }^{149}$.

\section{Targeted treatment for primary aldosteronism.}

In patients with FH-I, aldosterone production can be suppressed by treatment with exogenous glucocorticoids, which suppress ACTH production diminishing the stimulatory drive on the adrenal cortex. Hypertension can be efficiently controlled by the use of low doses of glucocorticoids, without requirement for complete suppression of ACTH and of hybrid gene expression ${ }^{4,37}$. Mineralocorticoid receptor antagonist may be added in the absence of complete normalization of blood pressure with glucocorticoid treatment ${ }^{4}{ }^{37}$. To date, no targeted treatment is available for patients with FH-II, FH-III and FH-IV; however, some evidence of therapeutic possibilities has come from mechanistic studies on cell systems. For instance, the discovery of recurrent mutations in genes encoding ion channels and ATPases in primary aldosteronism has opened new perspectives for targeted therapy for carriers of specific mutations ${ }^{109,110,117}$. In vitro studies showed that the pharmacology of mutant GIRK4 channels is distinct from wild-type channels and that they were blocked by calcium and sodium channel blockers, such as verapamil and amiloride ${ }^{109}$. In particular, high therapeutic doses of verapamil were able to specifically block GIRK4 channels carrying the recurrent p.Leu168Arg mutation $^{109}$. 
Macrolide antibiotics, in particular roxithromycin, are also able to selectively inhibit GIRK4 channels carrying the two most frequent mutations (p.Gly151Arg and p.Leu168Arg), with a decrease in $C Y P 11 B 2$ expression and aldosterone production in adrenocortical HAC15 cells 110. These results were confirmed in a study testing the effects of the macrolide clarithromycin on aldosterone synthesis and secretion in cells obtained from APA tissues with and without KCNJ5 mutations ${ }^{150}$. In a dose-dependent manner, clarithromycin reduced CYP11B2 expression and aldosterone secretion specifically in cells isolated ex vivo from $K C N J 5$ mutated $\mathrm{APA}^{150}$. These results suggest that the use of macrolides could be useful for noninvasive diagnosis and targeted treatment of APA with KCNJ5 mutations and for the treatment of patients with FH-III ${ }^{117}$. In addition to the inhibition of mutant GIRK4 with macrolides, calcium channel blockers might be used for patients with $C A C N A 1 H$ mutations in FH-IV or for patients with somatic $C A C N A 1 D$ mutations in APA, targeting the mutated calcium channels. In the same context, patients with PASNA seemed to well respond to the use of nifedipine for the treatment of hypertension and hypokalemia ${ }^{52,54}$.

Data on specific treatments for APA with somatic mutations, together with clinical studies evaluating the possibility of translating these findings to patient care, represent a great opportunity for the development of personalized treatments for a common endocrine form of arterial hypertension.

\section{Conclusions}

A large number of genetic and clinical studies have improved our knowledge of the pathogenesis and management of primary aldosteronism over the past few years (Figure 2). Genetic studies have been driven by the availability of high throughput genetic technologies. These technologies have enabled the discovery of genetic abnormalities in a large number of APA, and have also opened an entirely new field of research on the somatic genetics of aldosterone producing cellular structures of the normal adrenal cortex and in different types of 
primary aldosteronism. It seems that immunohistochemistry-guided NGS enables the identification of somatic mutations in the majority of patients with APA, which suggests that no further genes are involved in the disease, or if they were, they would account for a limited number of cases. Accumulation of APCC with somatic mutations has been reported in bilateral forms of primary aldosteronism; those results require replication to confirm that APCC are the sole cause of bilateral adrenal hyperplasia. The two pathogenic models of APA development, the APCC model and the two-hit model, are not mutually exclusive. Both mechanisms could be responsible for APA development depending on specific conditions, such as the adrenal microenvironment or genetic susceptibility. Most importantly, genetic studies have opened extremely interesting diagnostic and treatment opportunities. Surrogate biomarkers of the mutation status might enable simplification of the procedure for diagnosis and subtype identification of primary aldosteronism. Innovative therapies might be used to specifically target mutation carriers in cases in which surgery is not possible or in bilateral forms, using specific channel inhibitors.

In summary, primary aldosteronism is the most frequent form of secondary arterial hypertension. Appropriate treatment may cure patients and decrease cardiovascular risk. However, the diagnosis of primary aldosteronism is complex and the disease is largely underdiagnosed. New knowledge on the genetic basis of primary aldosteronism may unveil the pathophysiology of bilateral and familial forms and the mechanisms of APA development, allowing the development of new pharmacological approaches specifically targeting mutated proteins. 


\section{References}

1. Monticone, S. et al. Prevalence and Clinical Manifestations of Primary Aldosteronism Encountered in Primary Care Practice. J Am Coll Cardiol 69, 1811-1820 (2017).

2. Hannemann, A. \& Wallaschofski, H. Prevalence of primary aldosteronism in patient's cohorts and in population-based studies--a review of the current literature. Horm Metab Res 44, 157-62 (2012).

3. Brown, J.M. et al. The Unrecognized Prevalence of Primary Aldosteronism. Ann Intern Med (2020).

4. Funder, J.W. et al. The Management of Primary Aldosteronism: Case Detection, Diagnosis, and Treatment: An Endocrine Society Clinical Practice Guideline. J Clin Endocrinol Metab 101, 1889-916 (2016).

5. Mulatero, P. et al. Prevalence and characteristics of familial hyperaldosteronism: the PATOGEN study (Primary Aldosteronism in TOrino-GENetic forms). Hypertension 58, 797-803 (2011).

6. Lifton, R.P. et al. A chimaeric 11beta-hydroxylase aldosterone synthase gene causes glucocorticoid-remediable aldosteronism and human hypertension. Nature 355, 262265 (1992).

7. Choi, M. et al. $\mathrm{K}+$ channel mutations in adrenal aldosterone-producing adenomas and hereditary hypertension. Science 331, 768-72 (2011).

8. Scholl, U.I. et al. Recurrent gain of function mutation in calcium channel CACNA1H causes early-onset hypertension with primary aldosteronism. Elife 4, e06315 (2015).

9. Scholl, U.I. et al. CLCN2 chloride channel mutations in familial hyperaldosteronism type II. Nat Genet 50, 349-354 (2018).

10. Fernandes-Rosa, F.L. et al. A gain-of-function mutation in the CLCN2 chloride channel gene causes primary aldosteronism. Nat Genet 50, 355-361 (2018).

11. Rossier, B.C., Baker, M.E. \& Studer, R.A. Epithelial sodium transport and its control by aldosterone: the story of our internal environment revisited. Physiol Rev 95, 297-340 (2015).

12. Savard, S., Amar, L., Plouin, P.F. \& Steichen, O. Cardiovascular complications associated with primary aldosteronism: a controlled cross-sectional study. Hypertension 62, 331-6 (2013).

13. Monticone, S. et al. Cardiovascular events and target organ damage in primary aldosteronism compared with essential hypertension: a systematic review and metaanalysis. Lancet Diabetes Endocrinol 6, 41-50 (2018).

14. Milliez, P. et al. Evidence for an increased rate of cardiovascular events in patients with primary aldosteronism. J Am Coll Cardiol 45, 1243-8 (2005).

15. Rossi, G.P. et al. Primary aldosteronism: cardiovascular, renal and metabolic implications. Trends Endocrinol Metab 19, 88-90 (2008).

16. Rossi, G.P. et al. A prospective study of the prevalence of primary aldosteronism in 1,125 hypertensive patients. J Am Coll Cardiol 48, 2293-300 (2006).

17. Born-Frontsberg, E. et al. Cardiovascular and cerebrovascular comorbidities of hypokalemic and normokalemic primary aldosteronism: results of the German Conn's Registry. J Clin Endocrinol Metab 94, 1125-30 (2009).

18. Rossi, G.P. et al. Remodeling of the left ventricle in primary aldosteronism due to Conn's adenoma. Circulation 95, 1471-8. (1997).

19. Freel, E.M. et al. Demonstration of blood pressure-independent noninfarct myocardial fibrosis in primary aldosteronism: a cardiac magnetic resonance imaging study. Circ Cardiovasc Imaging 5, 740-7 (2012). 
20. Leopold, J.A. et al. Aldosterone impairs vascular reactivity by decreasing glucose-6phosphate dehydrogenase activity. Nat Med 13, 189-97 (2007).

21. Brown, N.J. Aldosterone and end-organ damage. Curr Opin Nephrol Hypertens 14, 235-41 (2005).

22. Calhoun, D.A. Hyperaldosteronism as a common cause of resistant hypertension. Annu Rev Med 64, 233-47 (2013).

23. Young, W.F., Jr. Diagnosis and treatment of primary aldosteronism: practical clinical perspectives. J Intern Med 285, 126-148 (2019).

24. Nishikawa, T. et al. Guidelines for the diagnosis and treatment of primary aldosteronism--the Japan Endocrine Society 2009. Endocr J 58, 711-21 (2011).

25. Eisenhofer, G. et al. Mass Spectrometry-Based Adrenal and Peripheral Venous Steroid Profiling for Subtyping Primary Aldosteronism. Clin Chem 62, 514-24 (2016).

26. Turcu, A.F. et al. Comprehensive Analysis of Steroid Biomarkers for Guiding Primary Aldosteronism Subtyping. Hypertension 75, 183-192 (2020).

27. Dekkers, T. et al. Adrenal vein sampling versus CT scan to determine treatment in primary aldosteronism: an outcome-based randomised diagnostic trial. Lancet Diabetes Endocrinol 4, 739-746 (2016).

28. Rossi, G.P. \& Funder, J.W. Adrenal Venous Sampling Versus Computed Tomographic Scan to Determine Treatment in Primary Aldosteronism (The SPARTACUS Trial): A Critique. Hypertension 69, 396-397 (2017).

29. Williams, T.A. et al. Computed Tomography and Adrenal Venous Sampling in the Diagnosis of Unilateral Primary Aldosteronism. Hypertension 72, 641-649 (2018).

30. Rossi, G.P. et al. Clinical Outcomes of 1625 Patients With Primary Aldosteronism Subtyped With Adrenal Vein Sampling. Hypertension 74, 800-808 (2019).

31. Stowasser, M. et al. Familial hyperaldosteronism type II: five families with a new variety of primary aldosteronism. Clin Exp Pharmacol Physiol 19, 319-22 (1992).

32. Geller, D.S. et al. A novel form of human mendelian hypertension featuring nonglucocorticoid-remediable aldosteronism. J Clin Endocrinol Metab 93, 3117-23 (2008).

33. Sutherland, D.J., Ruse, J.L. \& Laidlaw, J.C. Hypertension, increased aldosterone secretion and low plasma renin activity relieved by dexamethasone. Can Med Assoc J 95, 1109-19 (1966).

34. Halperin, F. \& Dluhy, R.G. Glucocorticoid-remediable aldosteronism. Endocrinol Metab Clin North Am 40, 333-41, viii (2011).

35. Pascoe, L. et al. Glucocorticoid-Suppressible Hyperaldosteronism Results from Hybrid Genes Created by Unequal Crossovers Between CYP11B1 and CYP11B2. Proc. Natl. Acad. Sci. - U.S.A. 89, 8327-8331 (1992).

36. Pascoe, L. et al. Glucocorticoid-suppressible hyperaldosteronism and adrenal tumors occurring in a single French pedigree. J Clin Invest 96, 2236-46 (1995).

37. Stowasser, M. \& Gordon, R.D. Primary aldosteronism: learning from the study of familial varieties. J Hypertens 18, 1165-76 (2000).

38. Mulatero, P. et al. Recombinant CYP11B genes encode enzymes that can catalyze conversion of 11-deoxycortisol to cortisol, 18-hydroxycortisol, and 18-oxocortisol. $J$ Clin Endocrinol Metab 83, 3996-4001 (1998).

39. Mulatero, P. et al. Diagnosis of glucocorticoid-remediable aldosteronism in primary aldosteronism: aldosterone response to dexamethasone and long polymerase chain reaction for chimeric gene. J Clin Endocrinol Metab 83, 2573-5 (1998).

40. Stowasser, M. \& Gordon, R.D. Familial hyperaldosteronism. J Steroid Biochem Mol Biol 78, 215-29 (2001). 
41. Medeau, V. et al. [Familial aspect of primary hyperaldosteronism: analysis of families compatible with primary hyperaldosteronism type 2]. Ann Endocrinol (Paris) 66, 2406 (2005).

42. Pallauf, A. et al. The prevalence of familial hyperaldosteronism in apparently sporadic primary aldosteronism in Germany: a single center experience. Horm Metab Res 44, 215-20 (2012).

43. Mulatero, P. et al. KCNJ5 mutations in European families with nonglucocorticoid remediable familial hyperaldosteronism. Hypertension 59, 235-40 (2012).

44. Daniil, G. et al. CACNA1H Mutations Are Associated With Different Forms of Primary Aldosteronism. EBioMedicine 13, 225-236 (2016).

45. Perez-Rivas, L.G., Williams, T.A. \& Reincke, M. Inherited Forms of Primary Hyperaldosteronism: New Genes, New Phenotypes and Proposition of A New Classification. Exp Clin Endocrinol Diabetes 127, 93-99 (2019).

46. Mulatero, P., Monticone, S., Rainey, W.E., Veglio, F. \& Williams, T.A. Role of KCNJ5 in familial and sporadic primary aldosteronism. Nat Rev Endocrinol 9, 104-12 (2013).

47. Scholl, U.I. et al. Hypertension with or without adrenal hyperplasia due to different inherited mutations in the potassium channel KCNJ5. Proc Natl Acad Sci U S A 109, 2533-8 (2012).

48. Monticone, S. et al. a Novel Y152C KCNJ5 mutation responsible for familial hyperaldosteronism type III. J Clin Endocrinol Metab 98, E1861-5 (2013).

49. Adachi, $\mathrm{M}$. et al. Discordant Genotype-Phenotype Correlation in Familial Hyperaldosteronism Type III with KCNJ5 Gene Mutation: A Patient Report and Review of the Literature. Horm Res Paediatr 82, 138-42 (2014).

50. Gomez-Sanchez, C.E. et al. Disordered zonal and cellular CYP11B2 enzyme expression in familial hyperaldosteronism type 3. Mol Cell Endocrinol 439, 74-80 (2017).

51. Tong, A. et al. A Novel Phenotype of Familial Hyperaldosteronism Type III: Concurrence of Aldosteronism and Cushing's Syndrome. J Clin Endocrinol Metab 101, 4290-4297 (2016).

52. Scholl, U.I. et al. Somatic and germline CACNA1D calcium channel mutations in aldosterone-producing adenomas and primary aldosteronism. Nat Genet 45, 1050-4 (2013).

53. Semenova, N.A., Ryzhkova, O.R., Strokova, T.V. \& Taran, N.N. [The third case report a patient with primary aldosteronism, seizures, and neurologic abnormalities (PASNA) syndrome de novo variant mutations in the CACNA1D gene]. Zh Nevrol Psikhiatr Im $S$ S Korsakova 118, 49-52 (2018).

54. De Mingo Alemany, M.C., Mifsud Grau, L., Moreno Macian, F., Ferrer Lorente, B. \& Leon Carinena, S. A de novo CACNA1D missense mutation in a patient with congenital hyperinsulinism, primary hyperaldosteronism and hypotonia. Channels (Austin) 14, 175-180 (2020).

55. Flanagan, S.E. et al. A CACNA1D mutation in a patient with persistent hyperinsulinaemic hypoglycaemia, heart defects, and severe hypotonia. Pediatr Diabetes 18, 320-323 (2017).

56. Dekkers, T. et al. Adrenal nodularity and somatic mutations in primary aldosteronism: one node is the culprit? J Clin Endocrinol Metab 99, E1341-51 (2014).

57. Omura, M., Sasano, H., Fujiwara, T., Yamaguchi, K. \& Nishikawa, T. Unique cases of unilateral hyperaldosteronemia due to multiple adrenocortical micronodules, which can only be detected by selective adrenal venous sampling. Metabolism 51, 350-5 (2002).

58. Monticone, S. et al. Immunohistochemical, genetic and clinical characterization of sporadic aldosterone-producing adenomas. Mol Cell Endocrinol 411, 146-54 (2015). 
59. Fernandes-Rosa, F.L. et al. Different Somatic Mutations in Multinodular Adrenals With Aldosterone-Producing Adenoma. Hypertension 66, 1014-22 (2015).

60. Yamazaki, Y. et al. Histopathological Classification of Cross-Sectional Image-Negative Hyperaldosteronism. J Clin Endocrinol Metab 102, 1182-1192 (2017).

61. Azizan, E.A. et al. Somatic mutations in ATP1A1 and CACNA1D underlie a common subtype of adrenal hypertension. Nat Genet 45, 1055-60 (2013).

62. Beuschlein, F. et al. Somatic mutations in ATP1A1 and ATP2B3 lead to aldosteroneproducing adenomas and secondary hypertension. Nat Genet 45, 440-4, 444e1-2 (2013).

63. Fernandes-Rosa, F.L. et al. Genetic spectrum and clinical correlates of somatic mutations in aldosterone-producing adenoma. Hypertension 64, 354-61 (2014).

64. Lenzini, L. et al. A Meta-Analysis of Somatic KCNJ5 K(+) Channel Mutations In 1636 Patients With an Aldosterone-Producing Adenoma. J Clin Endocrinol Metab 100, E1089-95 (2015).

65. Zennaro, M.C., Boulkroun, S. \& Fernandes-Rosa, F. Genetic Causes of Functional Adrenocortical Adenomas. Endocr Rev 38, 516-537 (2017).

66. Scholl, U.I. et al. Novel somatic mutations in primary hyperaldosteronism are related to the clinical, radiological and pathological phenotype. Clin Endocrinol (Oxf) 83, 779-89 (2015).

67. Akerstrom, T. et al. Activating mutations in CTNNB1 in aldosterone producing adenomas. Sci Rep 6, 19546 (2016).

68. Teo, A.E. et al. Pregnancy, Primary Aldosteronism, and Adrenal CTNNB1 Mutations. N Engl J Med 373, 1429-36 (2015).

69. Rhayem, Y. et al. PRKACA Somatic Mutations Are Rare Findings in AldosteroneProducing Adenomas. J Clin Endocrinol Metab 101, 3010-7 (2016).

70. Tadjine, M., Lampron, A., Ouadi, L. \& Bourdeau, I. Frequent mutations of beta-catenin gene in sporadic secreting adrenocortical adenomas. Clin Endocrinol (Oxf) 68, 264-70 (2008).

71. Tissier, F. et al. Mutations of beta-catenin in adrenocortical tumors: activation of the Wnt signaling pathway is a frequent event in both benign and malignant adrenocortical tumors. Cancer Res 65, 7622-7 (2005).

72. Beuschlein, F. et al. Constitutive activation of PKA catalytic subunit in adrenal Cushing's syndrome. N Engl J Med 370, 1019-28 (2014).

73. Cao, Y. et al. Activating hotspot L205R mutation in PRKACA and adrenal Cushing's syndrome. Science 344, 913-7 (2014).

74. Sato, Y. et al. Recurrent somatic mutations underlie corticotropin-independent Cushing's syndrome. Science 344, 917-20 (2014).

75. Goh, G. et al. Recurrent activating mutation in PRKACA in cortisol-producing adrenal tumors. Nat Genet 46, 613-7 (2014).

76. Nanba, K. et al. Targeted Molecular Characterization of Aldosterone-Producing Adenomas in White Americans. J Clin Endocrinol Metab 103, 3869-3876 (2018).

77. Nanba, K. et al. Genetic Characteristics of Aldosterone-Producing Adenomas in Blacks. Hypertension 73, 885-892 (2019).

78. De Sousa, K. et al. Genetic, Cellular, and Molecular Heterogeneity in Adrenals With Aldosterone-Producing Adenoma. Hypertension 75, 1034-1044 (2020).

79. Nishimoto, K. et al. Aldosterone-stimulating somatic gene mutations are common in normal adrenal glands. Proc Natl Acad Sci U S A 112, E4591-9 (2015).

80. Omata, K. et al. Cellular and Genetic Causes of Idiopathic Hyperaldosteronism. Hypertension 72, 874-880 (2018).

81. Spat, A. \& Hunyady, L. Control of aldosterone secretion: a model for convergence in cellular signaling pathways. Physiol Rev 84, 489-539 (2004). 
82. Oki, K., Plonczynski, M.W., Lam, M.L., Gomez-Sanchez, E.P. \& Gomez-Sanchez, C.E. The potassium channel, Kir3.4 participates in angiotensin II-stimulated aldosterone production by a human adrenocortical cell line. Endocrinology 153, 4328-35 (2012).

83. Murthy, M., Azizan, E.A., Brown, M.J. \& O'Shaughnessy, K.M. Characterization of a novel somatic KCNJ5 mutation delI157 in an aldosterone-producing adenoma. $J$ Hypertens 30, 1827-33 (2012).

84. Cheng, C.J. et al. Novel KCNJ5 mutations in sporadic aldosterone-producing adenoma reduce Kir3.4 membrane abundance. J Clin Endocrinol Metab 100, E155-63 (2015).

85. Yang, Y. et al. Primary Aldosteronism: KCNJ5 Mutations and Adrenocortical Cell Growth. Hypertension 74, 809-816 (2019).

86. Jentsch, T.J. \& Pusch, M. CLC Chloride Channels and Transporters: Structure, Function, Physiology, and Disease. Physiol Rev 98, 1493-1590 (2018).

87. Grunder, S., Thiemann, A., Pusch, M. \& Jentsch, T.J. Regions involved in the opening of CIC-2 chloride channel by voltage and cell volume. Nature 360, 759-62 (1992).

88. Dutta, R.K. et al. A somatic mutation in CLCN2 identified in a sporadic aldosteroneproducing adenoma. Eur J Endocrinol 181, K37-K41 (2019).

89. Goppner, C. et al. Pathogenesis of hypertension in a mouse model for human CLCN2 related hyperaldosteronism. Nat Commun 10, 4678 (2019).

90. Schewe, J. et al. Elevated aldosterone and blood pressure in a mouse model of familial hyperaldosteronism with ClC-2 mutation. Nat Commun 10, 5155 (2019).

91. Stindl, J. et al. Pathogenesis of Adrenal Aldosterone-Producing Adenomas Carrying Mutations of the $\mathrm{Na}(+) / \mathrm{K}(+)$-ATPase. Endocrinology 156, 4582-91 (2015).

92. Kaplan, J.H. Biochemistry of Na,K-ATPase. Annu Rev Biochem 71, 511-35 (2002).

93. Williams, T.A. et al. Somatic ATP1A1, ATP2B3, and KCNJ5 mutations in aldosteroneproducing adenomas. Hypertension 63, 188-95 (2014).

94. Zheng, F.F. et al. Clinical characteristics of somatic mutations in Chinese patients with aldosterone-producing adenoma. Hypertension 65, 622-8 (2015).

95. Akerstrom, T. et al. Novel somatic mutations and distinct molecular signature in aldosterone-producing adenomas. Endocr Relat Cancer 22, 735-44 (2015).

96. Einholm, A.P., Andersen, J.P. \& Vilsen, B. Importance of Leu99 in transmembrane segment M1 of the $\mathrm{Na}+, \mathrm{K}+-\mathrm{ATPase}$ in the binding and occlusion of $\mathrm{K}+$. J Biol Chem 282, 23854-66 (2007).

97. Dutta, R.K. et al. Complementary somatic mutations of KCNJ5, ATP1A1, and ATP2B3 in sporadic aldosterone producing adrenal adenomas. Endocr Relat Cancer 21, L1-4 (2014).

98. Tauber, P. et al. Cellular Pathophysiology of an Adrenal Adenoma-Associated Mutant of the Plasma Membrane Ca(2+)-ATPase ATP2B3. Endocrinology 157, 2489-99 (2016).

99. Nishimoto, K. et al. Case Report: Nodule Development From Subcapsular AldosteroneProducing Cell Clusters Causes Hyperaldosteronism. J Clin Endocrinol Metab 101, 69 (2016).

100. Nishimoto, K. et al. Adrenocortical zonation in humans under normal and pathological conditions. J Clin Endocrinol Metab 95, 2296-305 (2010).

101. Boulkroun, S. et al. Adrenal cortex remodeling and functional zona glomerulosa hyperplasia in primary aldosteronism. Hypertension 56, 885-92 (2010).

102. Nishimoto, K. et al. Immunohistochemistry of aldosterone synthase leads the way to the pathogenesis of primary aldosteronism. Mol Cell Endocrinol 441, 124-133 (2017).

103. Sun, N. et al. Mass Spectrometry Imaging Establishes 2 Distinct Metabolic Phenotypes of Aldosterone-Producing Cell Clusters in Primary Aldosteronism. Hypertension 75, 634-644 (2020). 
104. Boulkroun, S. et al. Aldosterone-producing adenoma formation in the adrenal cortex involves expression of stem/progenitor cell markers. Endocrinology 152, 4753-63 (2011).

105. Berthon, A. et al. WNT/beta-catenin signalling is activated in aldosterone-producing adenomas and controls aldosterone production. Hum Mol Genet 23, 889-905 (2014).

106. Enberg, U. et al. Postoperative differentiation between unilateral adrenal adenoma and bilateral adrenal hyperplasia in primary aldosteronism by mRNA expression of the gene CYP11B2. Eur J Endocrinol 151, 73-85 (2004).

107. Vouillarmet, J. et al. Aldosterone-Producing Adenoma With a Somatic KCNJ5 Mutation Revealing APC-Dependent Familial Adenomatous Polyposis. J Clin Endocrinol Metab 101, 3874-3878 (2016).

108. Stowasser, M. et al. Evidence for abnormal left ventricular structure and function in normotensive individuals with familial hyperaldosteronism type I. J Clin Endocrinol Metab 90, 5070-6 (2005).

109. Tauber, P. et al. Pharmacology and pathophysiology of mutated KCNJ5 found in adrenal aldosterone-producing adenomas. Endocrinology 155, 1353-62 (2014).

110. Scholl, U.I. et al. Macrolides selectively inhibit mutant KCNJ5 potassium channels that cause aldosterone-producing adenoma. J Clin Invest 127, 2739-2750 (2017).

111. Williams, T.A. et al. Genotype-Specific Steroid Profiles Associated With AldosteroneProducing Adenomas. Hypertension 67, 139-45 (2016).

112. Osswald, A. et al. Lack of influence of somatic mutations on steroid gradients during adrenal vein sampling in aldosterone-producing adenoma patients. Eur J Endocrinol 169, 657-63 (2013).

113. Kitamoto, T. et al. Clinical and Steroidogenic Characteristics of Aldosterone-Producing Adenomas With ATPase or CACNA1D Gene Mutations. J Clin Endocrinol Metab 101, 494-503 (2016).

114. Hattangady, N.G. et al. Mutated KCNJ5 activates the acute and chronic regulatory steps in aldosterone production. J Mol Endocrinol 57, 1-11 (2016).

115. Tezuka, Y. et al. 18-Oxocortisol Synthesis in Aldosterone-Producing Adrenocortical Adenoma and Significance of KCNJ5 Mutation Status. Hypertension 73, 1283-1290 (2019).

116. Murakami, M. et al. In situ metabolomics of aldosterone-producing adenomas. JCI Insight 4, e130356 (2019).

117. Maiolino, G. et al. Macrolides for KCNJ5-mutated aldosterone-producing adenoma (MAPA): design of a study for personalized diagnosis of primary aldosteronism. Blood Press 27, 200-205 (2018).

118. Catena, C. et al. Cardiovascular outcomes in patients with primary aldosteronism after treatment. Arch Intern Med 168, 80-5 (2008).

119. Reincke, M. et al. Observational study mortality in treated primary aldosteronism: the German Conn's registry. Hypertension 60, 618-24 (2012).

120. Wu, V.C. et al. Long term outcome of Aldosteronism after target treatments. Sci Rep $\mathbf{6}$, 32103 (2016).

121. Sukor, N., Kogovsek, C., Gordon, R.D., Robson, D. \& Stowasser, M. Improved quality of life, blood pressure, and biochemical status following laparoscopic adrenalectomy for unilateral primary aldosteronism. J Clin Endocrinol Metab 95, 1360-4 (2010).

122. Steichen, O., Zinzindohoue, F., Plouin, P.F. \& Amar, L. Outcomes of adrenalectomy in patients with unilateral primary aldosteronism: a review. Horm Metab Res 44, 221-7 (2012). 
123. Rossi, G.P. et al. Long-term control of arterial hypertension and regression of left ventricular hypertrophy with treatment of primary aldosteronism. Hypertension 62, 629 (2013).

124. Williams, T.A. et al. Outcomes after adrenalectomy for unilateral primary aldosteronism: an international consensus on outcome measures and analysis of remission rates in an international cohort. Lancet Diabetes Endocrinol 5, 689-699 (2017).

125. Chang, Y.H. et al. Surgery decreases the long-term incident stroke risk in patients with primary aldosteronism. Surgery 167, 367-377 (2020).

126. Rossi, G.P. et al. Adrenalectomy Lowers Incident Atrial Fibrillation in Primary Aldosteronism Patients at Long Term. Hypertension 71, 585-591 (2018).

127. Lin, Y.H. et al. Adrenalectomy improves increased carotid intima-media thickness and arterial stiffness in patients with aldosterone producing adenoma. Atherosclerosis 221, 154-9 (2012).

128. Lin, Y.H. et al. Adrenalectomy reverses myocardial fibrosis in patients with primary aldosteronism. J Hypertens 30, 1606-13 (2012).

129. Komada, H. et al. Insulin secretion and sensitivity before and after surgical treatment for aldosterone-producing adenoma. Diabetes Metab (2019).

130. Sonino, N. et al. Psychological assessment of primary aldosteronism: a controlled study. J Clin Endocrinol Metab 96, E878-83 (2011).

131. Apostolopoulou, K. et al. Gender differences in anxiety and depressive symptoms in patients with primary hyperaldosteronism: a cross-sectional study. World J Biol Psychiatry 15, 26-35 (2014).

132. Velema, M. et al. Quality of Life in Primary Aldosteronism: A Comparative Effectiveness Study of Adrenalectomy and Medical Treatment. J Clin Endocrinol Metab 103, 16-24 (2018).

133. Citton, M., Viel, G., Torresan, F., Rossi, G.P. \& Iacobone, M. Effect of unilateral adrenalectomy on the quality of life of patients with lateralized primary aldosteronism. BMC Surg 18, 105 (2019).

134. Ishidoya, S. et al. Changes in quality of life after laparoscopic adrenalectomy for patients with primary aldosteronism: Prospective 2-year longitudinal cohort study in a Japanese tertiary center. Int J Urol 26, 752-753 (2019).

135. Velema, M.S. et al. A disease-specific Quality of Life questionnaire for primary aldosteronism. Endocr Connect 8, 389-397 (2019).

136. Morisaki, M. et al. Predictors of Clinical Success After Surgery for Primary Aldosteronism in the Japanese Nationwide Cohort. J Endocr Soc 3, 2012-2022 (2019).

137. Vilela, L.A.P. et al. KCNJ5 Somatic Mutation Is a Predictor of Hypertension Remission After Adrenalectomy for Unilateral Primary Aldosteronism. J Clin Endocrinol Metab 104, 4695-4702 (2019).

138. Parthasarathy, H.K. et al. A double-blind, randomized study comparing the antihypertensive effect of eplerenone and spironolactone in patients with hypertension and evidence of primary aldosteronism. J Hypertens 29, 980-90 (2011).

139. Karagiannis, A. et al. Spironolactone versus eplerenone for the treatment of idiopathic hyperaldosteronism. Expert Opin Pharmacother 9, 509-15 (2008).

140. Rossi, G.P. Primary Aldosteronism: JACC State-of-the-Art Review. J Am Coll Cardiol 74, 2799-2811 (2019).

141. Jeunemaitre, X. et al. Efficacy and tolerance of spironolactone in essential hypertension. Am J Cardiol 60, 820-5 (1987).

142. de Gasparo, M. et al. Three new epoxy-spirolactone derivatives: characterization in vivo and in vitro. J Pharmacol Exp Ther 240, 650-6 (1987). 
143. Burgess, E.D. et al. Long-term safety and efficacy of the selective aldosterone blocker eplerenone in patients with essential hypertension. Clin Ther 25, 2388-404 (2003).

144. Capelli, I. et al. New mineralocorticoid receptor antagonists: update on their use in chronic kidney disease and heart failure. J Nephrol 33, 37-48 (2020).

145. Mulatero, P. et al. Long-term cardio- and cerebrovascular events in patients with primary aldosteronism. J Clin Endocrinol Metab 98, 4826-33 (2013).

146. Hundemer, G.L., Curhan, G.C., Yozamp, N., Wang, M. \& Vaidya, A. Cardiometabolic outcomes and mortality in medically treated primary aldosteronism: a retrospective cohort study. Lancet Diabetes Endocrinol 6, 51-59 (2018).

147. Hundemer, G.L., Curhan, G.C., Yozamp, N., Wang, M. \& Vaidya, A. Incidence of Atrial Fibrillation and Mineralocorticoid Receptor Activity in Patients With Medically and Surgically Treated Primary Aldosteronism. JAMA Cardiol 3, 768-774 (2018).

148. Hundemer, G.L., Curhan, G.C., Yozamp, N., Wang, M. \& Vaidya, A. Renal Outcomes in Medically and Surgically Treated Primary Aldosteronism. Hypertension 72, 658-666 (2018).

149. Vaidya, A., Mulatero, P., Baudrand, R. \& Adler, G.K. The Expanding Spectrum of Primary Aldosteronism: Implications for Diagnosis, Pathogenesis, and Treatment. Endocr Rev 39, 1057-1088 (2018).

150. Caroccia, B. et al. Macrolides Blunt Aldosterone Biosynthesis: A Proof-of-Concept Study in KCNJ5 Mutated Adenoma Cells Ex Vivo. Hypertension 70, 1238-1242 (2017).

151. Conn, J.W. Presidential address. I. Painting background. II. Primary aldosteronism, a new clinical syndrome. J Lab Clin Med 45, 3-17 (1955).

152. Conn, J.W. \& Louis, L.H. Primary aldosteronism: a new clinical entity. Trans Assoc Am Physicians 68, 215-31; discussion, 231-3 (1955).

153. Gordon, R.D. et al. Clinical and pathological diversity of primary aldosteronism, including a new familial variety. Clin Exp Pharmacol Physiol 18, 283-6 (1991).

154. Funder, J.W. et al. Case detection, diagnosis, and treatment of patients with primary aldosteronism: an endocrine society clinical practice guideline. J Clin Endocrinol Metab 93, 3266-81 (2008). 


\section{Acknowledgments}

The laboratory of Dr Maria-Christina Zennaro is supported through institutional funding from INSERM, by the Agence Nationale pour la Recherche (ANR-15-CE14-0017-03, ANR-18CE93-0003-01), the Fondation pour la Recherche Médicale (EQU201903007864) and by the H2020 project ENSAT-HT grant number 633983.

\section{Author contributions}

The authors contributed equally to all aspects of the article.

\section{Competing interests}

The authors declare no competing interests. 


\section{Figure 1 | Genetic and pathogenic mechanisms of familial hyperaldosteronism and}

targeted treatment. Familial hyperaldosteronism type I (FH-I) is due to unequal crossing-over between the $C Y P 11 B 1$ and $C Y P 11 B 2$ genes, resulting in the fusion of the regulatory sequences of $C Y P 11 B 1$ to the coding sequence of $C Y P 11 B 2$. This unequal crossing-over leads to ectopic aldosterone synthase expression in the zona fasciculata and stimulation of aldosterone production by ACTH. Targeted treatment is based on the use of glucocorticoids. FH-II is due to mutations in the CLCN2 gene, which encodes the chloride channel $\mathrm{ClC}-2$, resulting in an increased efflux of $\mathrm{Cl}^{-}$ions, leading to cell membrane depolarization, opening of voltage-gated$\mathrm{Ca}^{2+}$ channels and increased aldosterone production. No targeted treatments for FH-II are currently available. FH-III is due to mutations in the potassium channel GIRK4, encoded by $K C N J 5$. These mutations are responsible for a change in the channel selectivity, with a loss of $\mathrm{K}^{+}$selectivity and an increased $\mathrm{Na}^{+}$influx, leading to cell membrane depolarization, opening of voltage-gated-Ca ${ }^{2+}$ channels and increased aldosterone production. No targeted treatments for FH-III are available so far, but macrolides specifically block the mutated channels and might represent potential pharmacological options. Mutations in $C A C N A 1 H$, encoding the $\mathrm{Ca}^{2+}$ channel Cav3.2, are responsible for FH-IV. These mutations result in changes in calcium current properties, leading to an increased intracellular concentration of $\mathrm{Ca}^{2+}$ and aldosterone production. Specific calcium channel blockers acting on mutated Cav3.2 channels could be a useful development for patients with FH-IV.

Figure 2 Timeline of the major discoveries in the understanding of the genetic basis of primary aldosteronism. Clinical advances ${ }^{8,10,32,33,52,151-153}$ are shown in purple boxes, with key genetic discoveries ${ }^{6,7,52,61,62,70}$ highlighted in green boxes. The publication of guidelines ${ }^{4,154}$ is indicated in pink boxes. APA, aldosterone producing adenoma; FH, familial hyperaldosteronism. 
Key points

- Primary aldosteronism is the most frequent form of secondary and curable hypertension

- The condition is largely underdiagnosed, preventing patients from targeted treatment and prevention of cardiovascular complications

- Different genetic abnormalities have been identified in aldosterone producing adenoma and familial forms of the disease

- Most genetic abnormalities increase intracellular calcium signalling in the adrenal zona glomerulosa, increasing aldosterone production

- New approaches are currently developed to achieve more rapid and precise diagnosis of the condition and for more efficient targeted treatment

Table of contents text:

This Review outlines the latest understanding of the pathogenesis of primary aldosteronism. Current treatment options are also discussed, including the potential for targeted therapies.

\section{Glossary}

Autonomous aldosterone production: Aldosterone production which is is autonomous from its physiological regulators and inappropriate to the salt and volume status of the individual

Bilateral adrenal hyperplasia: One of the two major causes of primary aldosteronism, also called idiopathic hyperaldosteronism, in which aldosterone is produced autonomously from both adrenal glands. 
Essential hypertension: Arterial hypertension without known identifiable cause, defined after having excluded all forms secondary to a defined disease. It represents $85-95 \%$ of cases.

Aldosterone lateralization index: Value reflecting the production of aldosterone from one adrenal compared to the other, calculated by dividing the aldosterone to cortisol ratio in the dominant adrenal vein by the ratio in the non-dominant adrenal vein, measured by adrenal vein sampling.

Lateralized aldosterone production: Autonomous aldosterone production from one adrenal gland

Lateralized primary aldosteronism: Primary aldosteronism due to autonomous aldosterone production from one adrenal gland. 


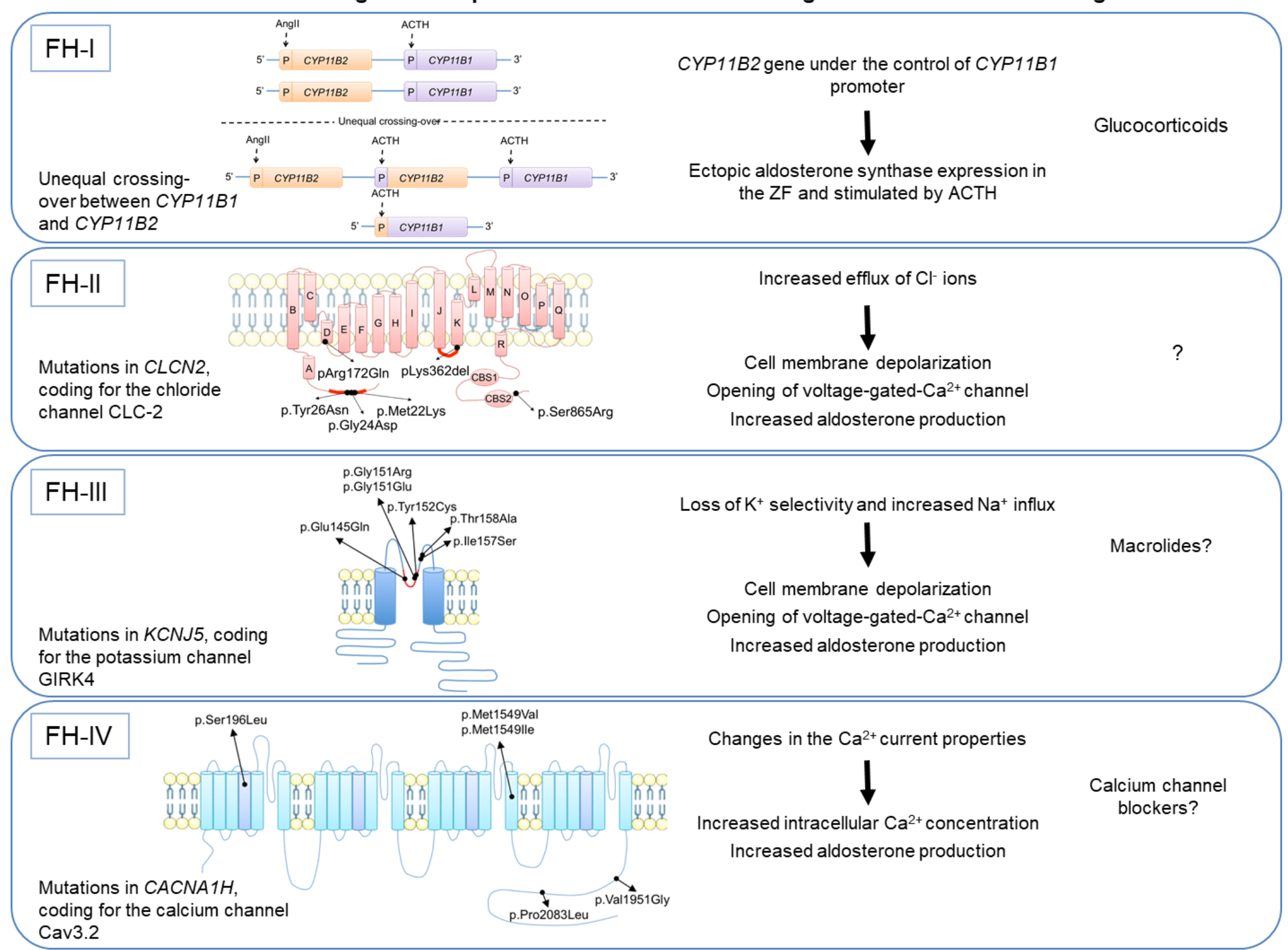

Figure 1 


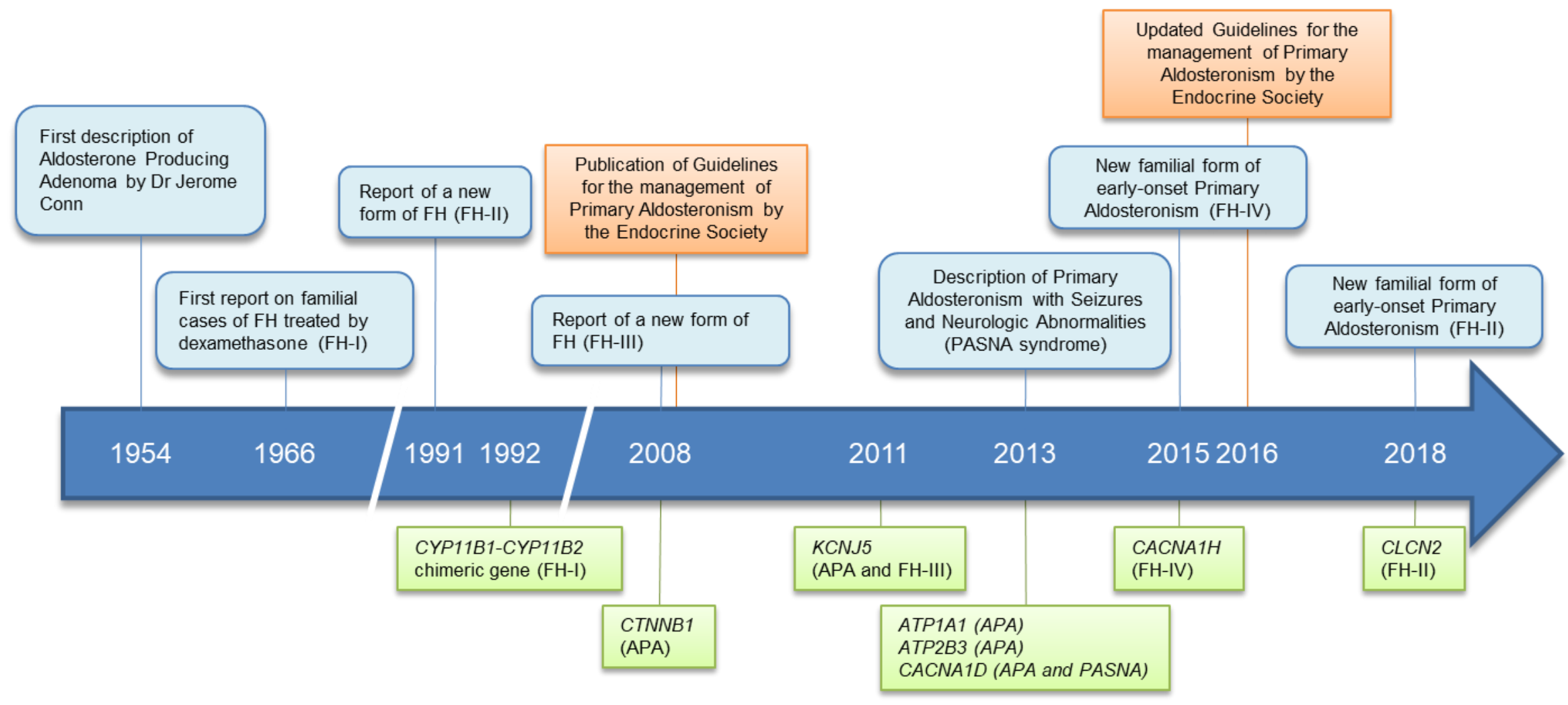

Figure 2 
